\title{
Minimal $k$-inflation in light of the conformal metric-affine geometry
}

\author{
Yusuke Mikura ${ }^{1,{ }^{*}}$ Yuichiro Tada $\odot,{ }^{1, \dagger}$ and Shuichiro Yokoyama ${ }^{2,3, *}$ \\ ${ }^{1}$ Department of Physics, Nagoya University, Nagoya 464-8602, Japan \\ ${ }^{2}$ Kobayashi Maskawa Institute, Nagoya University, Chikusa, Aichi 464-8602, Japan \\ ${ }^{3}$ Kavli IPMU (WPI), UTIAS, The University of Tokyo, Kashiwa, Chiba 277-8583, Japan
}

(Received 31 March 2021; accepted 30 April 2021; published 28 May 2021)

\begin{abstract}
We motivate a minimal realization of slow-roll $k$-inflation by incorporating the local conformal symmetry and the broken global $\mathrm{SO}(1,1)$ symmetry in the metric-affine geometry. With use of the metricaffine geometry where both the metric and the affine connection are treated as independent variables, the local conformal symmetry can be preserved in each term of the Lagrangian and thus higher derivatives of scalar fields can be easily added in a conformally invariant way. Predictions of this minimal slow-roll $k$-inflation, $n_{\mathrm{s}} \sim 0.96, r \sim 0.005$, and $c_{\mathrm{s}} \sim 0.03$, are not only consistent with current observational data but also have a prospect to be tested by forthcoming observations.
\end{abstract}

DOI: 10.1103/PhysRevD.103.L101303

\section{INTRODUCTION}

Nowadays cosmic inflation is considered as the standard paradigm for the early Universe [1-6]. Since the advent of its concept, a considerable number of inflationary models have been proposed for its realization from different motivations [7]. One typical scenario is that the accelerated expansion is driven by the potential energy of a scalar field, called inflaton. In particular, a class of $\alpha$ attractors attracts more and more attention of cosmologists because its predictions are in great agreement with observations of the cosmic microwave background (CMB) represented by the Planck Collaboration [8]. Besides its phenomenological success, an intriguing aspect lies in its guiding principles, the local conformal symmetry and slightly broken global symmetry. The former symmetry is an important concept in many physical contexts (see, e.g., Ref. [9]) and the latter one can be powerful to control fields' interactions. Indeed, successful representatives of the class, the $T$ model [10-14] and the Starobinsky model [1], can be derived from the viewpoint of the local conformal symmetry and the broken global $\mathrm{SO}(1,1)$ symmetry, where the inflaton can be recognized as the pseudo Nambu-Goldstone mode of the broken global symmetry.

\footnotetext{
*mikura.yusuke@e.mbox.nagoya-u.ac.jp

tada.yuichiro@e.mbox.nagoya-u.ac.jp

"shu@kmi.nagoya-u.ac.jp
}

Published by the American Physical Society under the terms of the Creative Commons Attribution 4.0 International license. Further distribution of this work must maintain attribution to the author(s) and the published article's title, journal citation, and DOI. Funded by SCOAP ${ }^{3}$.
Inflationary models in light of the local conformal symmetry, dubbed "conformal inflation," have been investigated mainly in the (pseudo-)Riemannian spacetime geometry, (see, e.g., Refs. [10,15-17] for a first concept and Refs. [18-22] for its phenomenology), where the metric $g$ is treated as an independent variable and the affine connection $\Gamma$ is determined only by the metric, i.e., it is usually the Levi-Civita connection. In this geometry, the Ricci scalar exhibits a nontrivial change as

$$
R(g) \rightarrow \tilde{R}(g)=\mathrm{e}^{2 \sigma(x)}\left(R(g)-6 \mathrm{e}^{\sigma(x)} \square \mathrm{e}^{-\sigma(x)}\right),
$$

under the local conformal transformation $g_{\mu \nu} \rightarrow \tilde{g}_{\mu \nu}=$ $\mathrm{e}^{-2 \sigma(x)} g_{\mu \nu}$ in the four-dimensional spacetime. Consequently, with a scalar field transforming as $S \rightarrow \tilde{S}=\mathrm{e}^{\sigma(x)} S$, the form of a locally conformal-invariant action is restricted as

$$
\begin{aligned}
\mathcal{L} & \supset \sqrt{-g}\left(\frac{1}{12} S^{2} R(g)-X(S)\right), \\
X(S) & :=-\frac{1}{2} g^{\mu \nu} \partial_{\mu} S \partial_{\nu} S,
\end{aligned}
$$

where a sign in front of the kinetic term $X(S)$ needs to be negative to cancel out extra $\sigma$ derivatives in Eq. (1) by the scalar kinetic term. The scalar $S$ thus behaves as a ghost in this case and should not appear in the physical spectrum.

From a geometrical viewpoint, one can use a generalized geometry called the metric-affine one in which the affine connection is treated a priori as an independent variable along with the metric (see, e.g., Refs. [23-28] and references therein). Applications of this geometry (or socalled Palatini formalism) to cosmology have gained increasing attention and a decent number of topics have 
been discussed in, e.g., Refs. [29-60] (see also Ref. [61] for a recent review). In this geometry, the Riemann tensor is a function only of the connection, being free from the metric, and thus can be covariant under the local conformal transformation. Furthermore, the naturally introduced vector, the nonmetricity $Q_{\mu}:=-g^{\alpha \beta} \nabla_{\mu} g_{\alpha \beta}$, plays the role of the conformal gauge field, which implements the covariant derivative $D_{\mu}:=\partial_{\mu}-\frac{1}{8} Q_{\mu}$ for scalars. ${ }^{1}$ Consequently, the conformal invariance can be preserved in each term of the Lagrangian in this geometry [71]. This independency is beneficial in various ways: e.g., it can be compatible with many kinds of extra symmetries and generalize the conformal class of potential-driven inflation as we discussed in the previous letter [72]. On the other kinetic side, the metric-affine geometry allows higher derivatives to be solely included in the Lagrangian in a conformally invariant way, being free from a specific structure (2) required in the Riemannian case.

It is widely known that such nonstandard kinetic terms can bring about inflation even if the potential term is absent, which we call kinetically driven inflation as a large class. The concept originates in $k$-inflation proposed in Refs. [73,74] and many models in this class such as the Dirac-Born-Infeld (DBI) model [75], the dilatonic ghost condensate [76,77], and $G$ inflation [78,79] have been later studied.

In this paper, we motivate a minimal realization of slowroll $k$-inflation by the local conformal symmetry and slightly broken global symmetry [specifically, $\mathrm{SO}(1,1)$ ] in the metric-affine geometry. We show that predictions of our minimal setup, the spectral index $n_{\mathrm{s}} \sim 0.96$, the tensorto-scalar ratio $r \sim 0.005$, and the sound speed $c_{\mathrm{s}} \sim 0.03$, are not only consistent with current observational data but also testable by future cosmological observations such as CMB, large-scale structures, and also stochastic gravitational waves. We adopt the natural unit $c=\hbar=1$ and the sign of the Minkowski metric is defined by $\eta_{\mu \nu}=\operatorname{diag}(-1,1,1,1)$ throughout.

\section{CONFORMAL METRIC-AFFINE GEOMETRY AND ITS COMPATIBILITY WITH HIGHER DERIVATIVES}

The metric-affine geometry treats both the metric and the affine connection as independent variables. In this geometry, the local conformal transformation is defined by the change of the metric, while the affine connection is left unaffected in contrast to the Riemannian one:

$$
g_{\mu \nu} \rightarrow \tilde{g}_{\mu \nu}=\mathrm{e}^{-2 \sigma(x)} g_{\mu \nu}, \quad \Gamma_{\mu \nu}^{\rho} \rightarrow \tilde{\Gamma}_{\mu \nu}^{\rho}=\Gamma_{\mu \nu}^{\rho} .
$$

\footnotetext{
${ }^{1}$ The nonmetricity is nondynamical in our case. Inflation with the dynamical nonmetricity known as the Weyl gauge field is discussed in Refs. [9,17,62-70].
}

Since the Ricci tensor $R_{\mu \nu}$ is a function only of the affine connection, it is obvious that the Ricci scalar transforms covariantly under the conformal transformation as

$$
R(g, \Gamma)=g^{\mu \nu} R_{\mu \nu}(\Gamma) \rightarrow R(\tilde{g}, \tilde{\Gamma})=\mathrm{e}^{2 \sigma(x)} R(g, \Gamma) .
$$

This covariant feature of the Ricci scalar points out that the nonminimal coupling term $\sqrt{-g} S^{2} R(g, \Gamma)$ is conformally invariant by itself without any help of the scalar kinetic term. The conformal invariance of the scalar kinetic term can be also accomplished with the help of the naturally introduced vector field, i.e., the nonmetricity [24]

$$
Q_{\mu}=g^{\alpha \beta} Q_{\mu \alpha \beta}:=-g^{\alpha \beta} \nabla_{\mu} g_{\alpha \beta} .
$$

Because it behaves as a gauge field associated with the conformal transformation,

$$
Q_{\mu} \rightarrow \tilde{Q}_{\mu}=Q_{\mu}+8 \partial_{\mu} \sigma,
$$

the covariant derivative for scalar fields can be defined as [71]

$$
D_{\mu} S:=\left(\partial_{\mu}-\frac{1}{8} Q_{\mu}\right) S,
$$

and one can easily show that the scalar kinetic term defined by $D_{\mu}$ transforms covariantly under the conformal transformation:

$$
\hat{X}(S):=-\frac{1}{2} g^{\mu \nu} D_{\mu} S D_{\nu} S \rightarrow \mathrm{e}^{4 \sigma(x)} \hat{X}(S) .
$$

This independency makes it possible to contain higher derivatives in the Lagrangian easily in a conformal-invariant way. A higher derivative term can be kept invariant if it is properly divided by the scalar as

$$
\mathcal{L} \supset \sqrt{-g}\left[\frac{C_{n}}{S^{4(n-1)}} \hat{X}^{n}\right],
$$

where $C_{n}(n=1,2, \ldots)$ is a dimensionless coupling constant.

Although higher derivatives can be easily compatible with the local conformal symmetry in the metric-affine geometry, such a scalar cannot be employed yet for, e.g., the inflation mechanism because it is removed from the theory by fixing the gauge symmetry. Hence one often introduces another scalar which plays a role in exhibiting the inflaton's dynamical degree of freedom. Upon adding another scalar, we can control their interactions with use of some global symmetry groups. For example, an $\mathrm{SO}(1,1)$ 
symmetry [10] between two scalars $\chi$ and $\phi$ allows us the following conformal Lagrangian up to $\hat{X}^{2}$ terms $^{2}$ :

$$
\begin{aligned}
\mathcal{L}= & \sqrt{-g}\left[\frac{\chi^{2}-\phi^{2}}{12} R(g, \Gamma)+\beta(\hat{X}(\chi)-\hat{X}(\phi))\right. \\
& \left.+\gamma^{4} \frac{(\hat{X}(\chi)-\hat{X}(\phi))^{2}}{\left(\chi^{2}-\phi^{2}\right)^{2}}\right]
\end{aligned}
$$

where $\beta$ and $\gamma$ are positive and dimensionless coupling constants. Thanks to the conformal symmetry, two scalars can be unified into one canonically normalized scalar $\varphi$ by gauge fixing. As a simple choice, it is convenient to take the so-called rapidity gauge $[10,12]$ defined through

$$
\begin{aligned}
& \chi=\sqrt{6} M_{\mathrm{Pl}} \cosh \frac{\varphi}{\sqrt{6} M_{\mathrm{Pl}}}, \\
& \phi=\sqrt{6} M_{\mathrm{Pl}} \sinh \frac{\varphi}{\sqrt{6} M_{\mathrm{Pl}}} .
\end{aligned}
$$

$M_{\mathrm{Pl}}$ is the reduced Planck mass. In this gauge, the nonminimal coupling term is fixed as the Einstein-Hilbert term and the Lagrangian is reduced to

$$
\begin{aligned}
\mathcal{L}= & \frac{M_{\mathrm{Pl}}^{2}}{2} R-\beta X(\varphi)-\frac{3 M_{\mathrm{Pl}}^{2}}{64} \beta Q^{\mu} Q_{\mu} \\
& +\frac{\gamma^{4}}{36 M_{\mathrm{Pl}}^{4}}\left(X^{2}(\varphi)+\frac{3 M_{\mathrm{Pl}}^{2}}{32} Q^{\mu} Q_{\mu} X(\varphi)\right. \\
& \left.+\frac{9 M_{\mathrm{Pl}}^{4}}{64 \cdot 64}\left(Q^{\mu} Q_{\mu}\right)^{2}\right),
\end{aligned}
$$

where $X(\varphi)=-\partial_{\mu} \varphi \partial^{\mu} \varphi / 2$ is the kinetic term constructed with a usual derivative. We note that the Ricci scalar is free from the nonmetricity $Q_{\mu}$ (see, e.g., Refs. [33,71]), and then its stationary solutions are determined only by explicit $Q_{\mu}$ terms in the action. One trivial solution is found as $Q_{\mu}=0,{ }^{3}$ which leads to

$$
\mathcal{L}=\frac{M_{\mathrm{Pl}}^{2}}{2} R-\beta X(\varphi)+\frac{\gamma^{4}}{36 M_{\mathrm{Pl}}^{4}} X^{2}(\varphi)
$$

This is nothing but a minimal realization of the so-called $k$ inflation $[73,74]$. The inflaton $\varphi$ realizes the kinetically driven de Sitter universe with the stationary momentum of

\footnotetext{
${ }^{2}$ The potential term $\frac{\lambda}{4}\left(\chi^{2}-\phi^{2}\right)^{2}$ investigated in our previous work [72] is also allowed by this symmetry, but we neglect it for simplicity in this work.

${ }^{3}$ The other nontrivial solution is $Q^{\mu} Q_{\mu}=\frac{64}{\gamma^{4}}\left(6 M_{\mathrm{Pl}}^{2} \beta-\frac{\gamma^{4}}{3 M_{\mathrm{Pl}}^{2}} X(\varphi)\right)$ and leads to $\mathcal{L}=\frac{M_{\mathrm{Pl}}^{2}}{2} R-9 M_{\mathrm{Pl}}^{4} \frac{\beta^{2}}{\gamma^{4}}$. We leave this nontrivial branch for a future issue. At least the attractor stability of the trivial branch (13) is confirmed [73,74].
}

$P(X)=-\beta X+\frac{\gamma^{4}}{36 M_{\mathrm{Pl}}^{4}} X^{2}$, i.e., $X=\dot{\varphi}^{2} / 2=\frac{18 \beta M_{\mathrm{P}}^{4}}{\gamma^{4}}$ at the background level.

Inflation cannot end if both $\beta$ and $\gamma$ are constant. Then we explicitly break the $\mathrm{SO}(1,1)$ symmetry by replacing $\beta$ with $\beta F(\phi / \chi)$, some function of $\phi / \chi=\tanh \frac{\varphi}{\sqrt{6} M_{\mathrm{Pl}}}$ which uniquely preserves the local conformal symmetry. The $\mathrm{SO}(1,1)$ symmetry is beneficial because it is restored in the large field limit $\varphi \rightarrow \pm \infty$. On the other hand, its explicit breaking naturally introduces the end of inflation. Assuming $F(x)$ is odd, $P(X)$ has a nontrivial minimum $X=\frac{18 \beta F M_{\mathrm{Pl}}^{4}}{\gamma^{4}}$ for $F>0$ corresponding to the de Sitter phase, while such a minimum is not realized for $F<0$ because $X=\dot{\varphi}^{2} / 2$ must be non-negative. Thus, the graceful exit can be dynamically realized around the symmetry breaking point $\varphi \sim 0$.

\section{MINIMAL SLOW-ROLL $\boldsymbol{k}$-INFLATION}

Thanks to the local conformal and broken global $\mathrm{SO}(1,1)$ symmetries, a flipping kinetic term of a hyperbolic-tangent form is naturally introduced. ${ }^{4}$ Such a Lagrangian is summarized as

$$
\begin{aligned}
\mathcal{L} & =\sqrt{-g}\left[\frac{M_{\mathrm{Pl}}^{2}}{2} R-K(\Phi) X(\Phi)+\frac{1}{M_{\mathrm{Pl}}^{4}} X^{2}(\Phi)\right], \\
K(\Phi) & =\frac{6 \beta}{\gamma^{2}} F\left(\tanh \left(\frac{\Phi}{\gamma M_{\mathrm{Pl}}}\right)\right),
\end{aligned}
$$

by field redefinition $\Phi:=\frac{\gamma}{\sqrt{6}} \varphi$. Now the gravitational sector is reduced to the ordinary Einstein-Hilbert one and there remains no difference between the Riemannian and metricaffine approaches. Thus it is a minimal realization of the original slow-roll $k$-inflation model $[73,74]$ with a hyperbolic-tangent kinetic term. Here we investigate its phenomenology as inflation, following Ref. [73].

At the background level, inflaton's pressure and energy density, $P$ and $\rho$, are given by

$$
P=-K(\Phi) X+\frac{1}{M_{\mathrm{Pl}}^{4}} X^{2}, \quad \rho=2 X P_{X}-P,
$$

where $P_{X}=\partial P / \partial X$. The master equation of evolution is obtained as

$$
\dot{\rho}=-\frac{\sqrt{3}}{M_{\mathrm{Pl}}} \sqrt{\rho}(\rho+P) \propto P_{X} .
$$

If $K(\Phi)$ is positive and its $\Phi$ dependence can be neglected, as we have mentioned, then we have a stationary solution

\footnotetext{
${ }^{4}$ The graceful exit making use of the hyperbolic-tangent switching of $X$ term itself is mentioned in Ref. [78] in the context of $G$ inflation and its gravitational reheating is discussed in Ref. [80].
} 
$\bar{P}_{X}:=\left.P_{X}\right|_{X=\bar{X}}=0$ with $\bar{X}=\dot{\bar{\Phi}}^{2} / 2=K M_{\mathrm{Pl}}^{4} / 2$ which gives the de Sitter universe $(\dot{\rho}=0)$. Here and hereafter barred quantities denote this de Sitter limit solution.

In realistic dynamics, $X$ however deviates from $\bar{X}$ due to the $\Phi$ dependence of $K(\Phi)$. Corresponding to the slow-roll expansion in ordinary inflation models, one can expand the dynamics with respect to this deviation $\delta X:=X-\bar{X}$ $[73,74]$. In fact, the master equation (16) indicates that the time dependence of the energy density dictating the slow-roll parameter $\epsilon=-\dot{H} / H^{2}$ is an $\mathcal{O}(\delta X)$ quantity as

$$
\dot{\rho}=-\left.\frac{\sqrt{3}}{M_{\mathrm{Pl}}} \sqrt{\rho}(\rho+P)\right|_{X=\bar{X}+\delta X} \simeq-\sqrt{3} K^{2} M_{\mathrm{Pl}} \delta X,
$$

at linear order in $\delta X$. On the other hand, one can express it in terms of $K$ 's time derivative via the leading form $\bar{\rho}=K^{2} M_{\mathrm{Pl}}^{4} / 4$ as

$$
\dot{\rho} \simeq \frac{K K_{\Phi} \dot{\bar{\Phi}} M_{\mathrm{Pl}}^{4}}{2},
$$

where $K_{\Phi}=\partial K / \partial \Phi$. Hence, as expected, $K$ 's time derivative representing the deviation from the exact de Sitter universe is also an $\mathcal{O}(\delta X)$ quantity as

$$
\delta X \simeq-\operatorname{sign}(\dot{\bar{\Phi}}) \frac{K_{\Phi} M_{\mathrm{Pl}}^{5}}{2 \sqrt{3 K}} .
$$

In order not to violate the null energy condition, i.e., $\rho+P>0, \delta X$ should be positive. Once $\delta X$ is expressed in $K$ 's derivatives, slow-roll parameters $\epsilon, \eta$, and $s$, and the sound speed $c_{\mathrm{s}}$ can be obtained as

$$
\left\{\begin{array}{l}
\epsilon:=-\frac{\dot{H}}{H^{2}} \simeq-\operatorname{sign}(\dot{\bar{\Phi}}) \frac{6 M_{\mathrm{Pl}}}{\sqrt{3}} \frac{K_{\Phi}}{K^{3 / 2}} \\
\eta:=\frac{\dot{\epsilon}}{H \epsilon} \simeq \operatorname{sign}(\dot{\bar{\Phi}}) 2 \sqrt{3} M_{\mathrm{Pl}}\left(\frac{K_{\Phi \Phi}}{K^{1 / 2} K_{\Phi}}-\frac{3}{2} \frac{K_{\Phi}}{K^{3 / 2}}\right), \\
c_{\mathrm{S}}^{2}:=\frac{P_{X}}{P_{X}+2 X P_{X X}} \simeq \frac{1}{12} \epsilon \\
s:=\frac{\dot{c}_{\mathrm{s}}}{H c_{\mathrm{s}}} \simeq \frac{1}{2} \eta
\end{array}\right.
$$

The primary cosmological observables, the spectral index $n_{\mathrm{s}}$ and the tensor-to-scalar ratio $r$, are expressed with use of these parameters as [74,81]

$n_{\mathrm{s}}-1:=\frac{\mathrm{d} \log \mathcal{P}_{\zeta}}{\mathrm{d} \log k} \simeq-2 \epsilon-\eta-s, \quad r:=\frac{\mathcal{P}_{h}}{\mathcal{P}_{\zeta}} \simeq 16 c_{\mathrm{s}} \epsilon$,

where $\mathcal{P}_{\zeta}$ and $\mathcal{P}_{h}$ denote the dimensionless power spectra of curvature and tensor perturbations, respectively. In addition, in such a kinetically driven inflation, $c_{s} \ll 1$ can be achieved and it predicts the large non-Gaussianity which can be observed as the so-called equilateral shape in the bispectrum of the primordial curvature perturbations: $f_{\mathrm{NL}}^{\mathrm{eq}} \sim 1 / c_{\mathrm{s}}^{2}$ (see, e.g., Ref. [81]). Through the identity $\mathrm{d} \Phi / \mathrm{d} N=-\dot{\Phi} / H$, the backward $e$-folds $N$ from the end of inflation can be also obtained as

$$
N:=\log \left(\frac{a_{\text {end }}}{a}\right) \simeq \frac{-\operatorname{sign}(\dot{\bar{\Phi}})}{2 \sqrt{3} M_{\mathrm{Pl}}} \int_{\bar{\Phi}_{\text {end }}}^{\bar{\Phi}} \sqrt{K} \mathrm{~d} \Phi,
$$

where $\bar{\Phi}_{\text {end }}$ denotes the field value at the end of inflation, i.e., $\epsilon=1$.

\section{LARGE $\beta$ LIMIT}

As we want to see the deviation from the exact de Sitter universe in this model, let us focus on the small field dynamics $\Phi \ll \gamma M_{\mathrm{Pl}}$ where $\tanh \left(\Phi / \gamma M_{\mathrm{Pl}}\right)$ can be approximated by $\Phi / \gamma M_{\mathrm{Pl}}$. This is actually accomplished in the large $\beta$ limit and we will see that the corresponding inflationary predictions are currently consistent with and testable in the future by cosmological observations.

Adopting the simplest form $F(x)=x$ for the symmetry breaking part, we hence approximate $K(\Phi)$ as

$$
K(\Phi) \sim \frac{6 \beta}{\gamma^{3}} \frac{\Phi}{M_{\mathrm{Pl}}} .
$$

With this simple assumption, the backward $e$-folds (22) and slow-roll parameters (20) are easily obtained as

$$
\frac{\bar{\Phi}}{\gamma M_{\mathrm{Pl}}}=\left(\frac{9 N^{2}}{2 \beta}\right)^{1 / 3}
$$

and

$n_{\mathrm{s}}-1 \simeq-\frac{17}{6 N}, \quad r \simeq \frac{16 \sqrt{2}}{9} \frac{1}{N^{3 / 2}}, \quad c_{\mathrm{s}} \simeq \sqrt{\frac{1}{18 N}}$.

Here we ignore $\bar{\Phi}_{\text {end }}$. The relation (24) in fact indicates that the small field assumption $\Phi \ll \gamma M_{\mathrm{Pl}}$ is equivalent to the condition on $\beta$ as $\beta \gg \frac{9}{2} N^{2}$. Note that the remained parameter $\gamma$ appears as a combination $\beta / \gamma^{3}$ in the power spectrum of the curvature perturbation given by

$\mathcal{P}_{\zeta}=\frac{1}{2 c_{\mathrm{s}} \epsilon M_{\mathrm{Pl}}^{2}}\left(\frac{H}{2 \pi}\right)^{2} \simeq \frac{81 \cdot 3^{1 / 3}}{16 \cdot 2^{1 / 6} \pi^{2}}\left(\frac{\beta}{\gamma^{3}}\right)^{4 / 3} N^{17 / 6}$.

Thus, for each value of $\beta, \gamma$ should be fixed to be consistent with the $\mathrm{CMB}$ observation $\mathcal{P}_{\zeta} \simeq 2.1 \times 10^{-9}$ [8].

The energy and pressure density after the end of inflation in our model can be well approximated as $\rho \simeq P \simeq-K X$ since the square of the kinetic term becomes negligibly small, and the effective equation-of-state parameter becomes $w:=P / \rho \simeq 1$, which phase is called "kination." The energy density and the Hubble scale in the kination epoch evolve as $\rho \propto a^{-6}$ and $H^{-1} \propto a^{3}$, respectively, whereas these evolve as $\rho \propto a^{-3}$ and $H^{-1} \propto a^{3 / 2}$ in the ordinary inflaton oscillation phase (matter domination). 

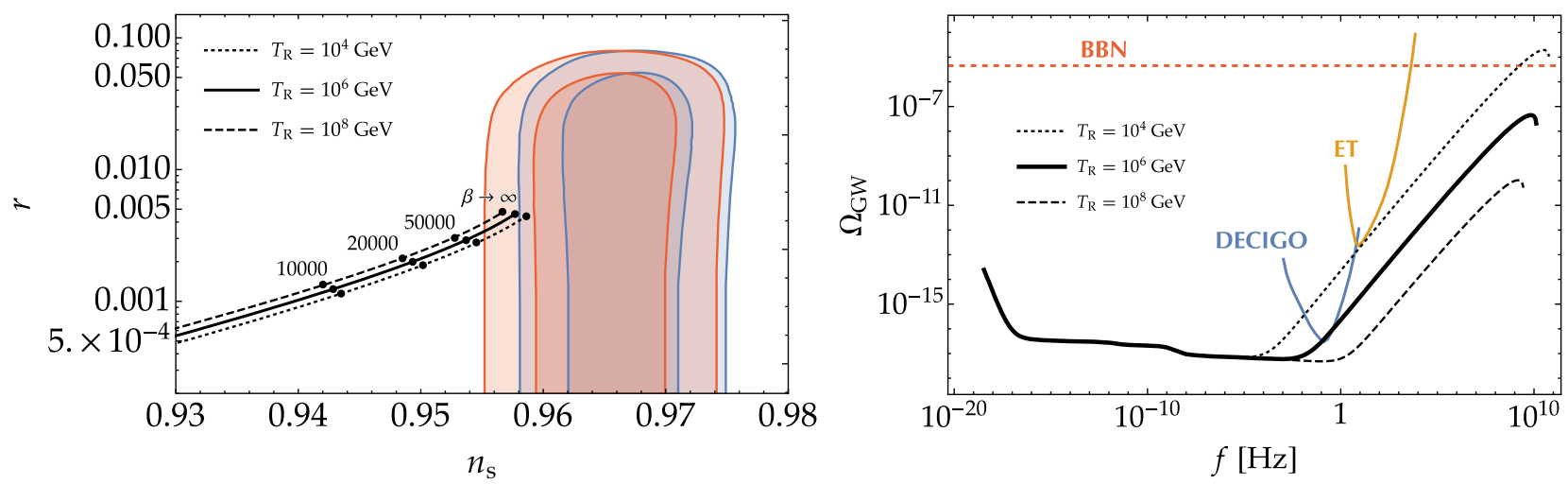

FIG. 1. Left: predictions for the spectral index $n_{\mathrm{s}}$ and the tensor-to-scalar ratio $r$ with varying the kinetic coefficient $\beta$ and reheating temperature $T_{\mathrm{R}}$. The black dotted, thick, and dashed lines correspond with $T_{\mathrm{R}}=10^{4}, 10^{6}$, and $10^{8} \mathrm{GeV}$, respectively, while the black points represent $\beta$ 's value of 10000, 20000, 50000, and the large $\beta$ limit (25) from left to right. We also plot the $1 \sigma$ and $2 \sigma$ constraints on the pivot scale $k=0.002 \mathrm{Mpc}^{-1}$ from Planck TT, TE, EE + lowE + lensing + BK15 (red lines) and Planck TT, TE, EE + lowE + lensing + BK15 + BAO (blue lines) [8]. Right: the current density parameter of stochastic gravitational waves with respect to the reheating temperature $T_{\mathrm{R}}$. Similarly to the left panel, the black dotted, thick, and dashed lines correspond with $T_{\mathrm{R}}=10^{4}, 10^{6}$, and $10^{8} \mathrm{GeV}$. The red dotted line indicates the BBN upper bound [88], while the blue and orange lines exhibit the future sensitivity prospects by DECIGO and Einstein Telescope observational projects (taken from Ref. [89]).

Therefore the correspondence between the perturbation scale $k_{*}$ and the backward $e$-folds from the end of inflation is altered from the ordinary case, and assuming the instant transition at each phase of the Universe, one finds the following formula:

$$
\begin{aligned}
N= & 66-\ln \left(\frac{k_{*}}{0.002 \mathrm{Mpc}^{-1}}\right)-\frac{1}{3} \ln \left(\frac{T_{\mathrm{R}}}{10^{6} \mathrm{GeV}}\right) \\
& -\frac{1}{6} \ln \left(\frac{g_{*}\left(T_{\mathrm{R}}\right)}{106.75}\right)+\frac{1}{3} \ln \left(\frac{H_{*}}{H_{\mathrm{f}}}\right)+\frac{1}{3} \ln \left(\frac{r_{*}}{0.005}\right) .
\end{aligned}
$$

Here the reheating temperature $T_{\mathrm{R}}$ denotes the radiation temperature at the time when the radiation energy density becomes comparable to the inflaton's one. $g_{*}$ stands for the effective degrees of freedom for energy density and we approximate it to be almost equal to ones for entropy density at $T=T_{\mathrm{R}} . H_{*}$ and $r_{*}$ are the Hubble parameter and the tensor-to-scalar ratio corresponding to the considered scale $k_{*}$, respectively. $H_{\mathrm{f}}$ is the Hubble parameter at the end of inflation. We adopt the standard values for $g_{*}$ at the matter-radiation equality and the current matter density parameter $\Omega_{\mathrm{m}}$ as $g_{*}\left(T_{\text {eq }}\right)=3.38$ and $\Omega_{\mathrm{m}} h^{2}=0.143$ with the normalized Hubble constant $h=H_{0} /(100 \mathrm{~km} / \mathrm{s} / \mathrm{Mpc})$, respectively [82].

The left panel of Fig. 1 shows our numerical predictions on $n_{\mathrm{s}}$ and $r$ without the linear approximation of $\tanh \left(\Phi / \gamma M_{\mathrm{Pl}}\right)$ in terms of the reheating temperature, varying $\beta$ (and $\gamma$ for a proper $\mathcal{P}_{\zeta}$ ). In the large limit of $\beta$, which well satisfies a condition $\beta \gg \frac{9}{2} N^{2}$, one confirms that predictions converge to the analytic formula (25). Predictions with large $\beta$ can be consistent with the
Planck 2018's $2 \sigma$ constraints $[8,83]$. In particular, the tensor-to-scalar ratio $r \sim 0.005$ is sizable enough for a future test by CMB observations such as LiteBIRD [84] and CMB-S4 $[85,86]$. One also finds, from the analytic formula (25), the predicted sound speed $c_{\mathrm{s}} \sim 0.03$ is consistently large with the $2 \sigma$ allowed value $c_{\mathrm{s}} \geq 0.021$ [83] from the non-Gaussianity on CMB, but characteristically small enough to be tested by, e.g., future galaxy observations [87].

\section{REHEATING AND GRAVITATIONAL WAVES}

As can be seen in the left panel of Fig. 1, the lower reheating temperature makes the $\left(n_{\mathrm{s}}, r\right)$ prediction more consistent with the $\mathrm{CMB}$ observations. As a possible reheating scenario in the kination phase, the so-called gravitational reheating $[90,91]$ has been widely discussed, in which a part of the inflaton's energy is transferred into the radiation through the sudden change of the Universe's expansion law between the inflation and kination eras. Contrary to the ordinary reheating scenario [92], the inflaton need not completely decay into the radiation because the inflaton's energy decreases as $\rho_{\varphi} \propto a^{-6}$, i.e., faster than radiational one $\rho_{\mathrm{r}} \propto a^{-4}$. Thus the radiation can dominate the Universe sooner or later. Consequently, the reheating temperature tends to be low in this scenario, and it is roughly given by $T_{\mathrm{R}} \sim H_{\mathrm{f}}^{2} / M_{\mathrm{Pl}}$ (see, e.g., Ref. [93]). In our model, it corresponds with $T_{\mathrm{R}} \sim 10^{6} \mathrm{GeV}$ and we treat it as a fiducial value, though we keep allowing lower or higher reheating temperatures as a free parameter because it may depend on what kind of particles were produced and also how the transition from the inflationary phase to the 
kination proceeds $[80,94]$. It also includes the other possibilities of reheating mechanisms [95]. ${ }^{5}$

The reheating temperature could be revealed by the stochastic gravitational waves [96]. Some random gravitational waves are produced as tensor perturbations on superhorizon scales during inflation as well as scalar perturbations. After their horizon reentry, they behave as freely propagating radiation, and their relative energy density compared to the background inflaton can then grow as $\rho_{\mathrm{GW}} / \rho_{\varphi} \propto a^{2}$ during the kination era. Therefore, for a lower reheating temperature or a longer kination phase, our inflation model predicts a larger stochastic gravitational wave amplitude [95,97-102]. In the right panel of Fig. 1, we show the prediction on the current density parameter $\Omega_{\mathrm{GW}}$ in terms of $T_{\mathrm{R}}$ as well as sensitivity prospects of DECIGO [103,104] and Einstein Telescope projects [105]. We also note that the gravitational wave amplitude is constrained from above not to spoil the successful big bang nucleosynthesis (BBN) [88,106], whose bound is exhibited by the red dotted line. One finds, for $10^{5} \mathrm{GeV} \lesssim T_{\mathrm{R}} \lesssim 10^{6} \mathrm{GeV}$, the predicted gravitational waves can evade the BBN constraint and can be tested by DECIGO.

\section{CONCLUSIONS}

In this paper, we investigated a minimal realization of slow-roll $k$-inflation (14) that arose from the Lagrangian up

\footnotetext{
${ }^{5}$ Our inflaton is a pseudo Nambu-Goldstone boson of the $\mathrm{SO}(1,1)$ symmetry, so basically it only has derivative couplings to matter fields. The efficiency of reheating is hence expected not to be large anyway.
}

to quadratic kinetic terms with the slightly broken global $\mathrm{SO}(1,1)$ symmetry in the conformal metric-affine geometry. The left panel of Fig. 1 shows the numerical predictions of the spectral index $n_{\mathrm{s}}$ and the tensor-to-scalar ratio $r$ with varying the coefficient $\beta$. We found that the observational predictions on the spectral index $n_{\mathrm{s}}$, the tensor-to-scalar ratio $r$, and the sound speed $c_{\mathrm{s}}$, converge in the large $\beta$ limit [i.e., $K(\Phi) \sim 6 \beta \Phi /\left(\gamma^{3} M_{\mathrm{Pl}}\right)$ as shown in Eq. (23)] and become consistent with current observational data, particularly for lower reheating temperature. The intriguing aspect of our model is its testability by the forthcoming advancement of cosmological observations. The predicted tensorto-scalar ratio $r \sim 0.005$ is sizable enough to be checked by future CMB observation plans such as LiteBIRD [84] and CMB-S4 $[85,86]$. In addition to the tensor-to-scalar ratio, the relatively small sound speed $c_{\mathrm{s}} \sim 0.03$ will leave a characteristic non-Gaussianity on primordial perturbations, which is particularly beneficial to distinguish among inflationary models. The detailed study on the non-Gaussianity by using galaxies [87] can potentially test our model as constraints on the sound speed get tighter. Furthermore, our model has also a prospect to be tested by future space-based gravitational wave detectors represented by DECIGO as depicted in the right panel of Fig. 1 since the kination epoch intensifies the amplitude of stochastic gravitational waves at high frequency.

\section{ACKNOWLEDGMENTS}

Y. T. is supported by JSPS KAKENHI Grants No. JP18J01992 and No. JP19K14707. S. Y. is supported by JSPS Grant-in-Aid for Scientific Research (B) No. JP20H01932 and (C) No. JP20K03968.
[1] A. A. Starobinsky, A new type of isotropic cosmological models without singularity, Adv. Ser. Astrophys. Cosmol. 3, 130 (1987).

[2] K. Sato, First order phase transition of a vacuum and expansion of the universe, Mon. Not. R. Astron. Soc. 195, 467 (1981).

[3] A. H. Guth, The inflationary universe: A possible solution to the horizon and flatness problems, Phys. Rev. D 23, 347 (1981).

[4] A. D. Linde, A new inflationary universe scenario: A possible solution of the horizon, flatness, homogeneity, isotropy and primordial monopole problems, Phys. Lett. 108B, 389 (1982).

[5] A. Albrecht and P. J. Steinhardt, Cosmology for Grand Unified Theories with Radiatively Induced Symmetry Breaking, Phys. Rev. Lett. 48, 1220 (1982).

[6] A. D. Linde, Chaotic inflation, Phys. Lett. 129B, 177 (1983).
[7] D. H. Lyth and A. Riotto, Particle physics models of inflation and the cosmological density perturbation, Phys. Rep. 314, 1 (1999).

[8] Y. Akrami et al. (Planck Collaboration), Planck 2018 results. X. Constraints on inflation, Astron. Astrophys. 641, A10 (2020).

[9] I. Bars, P. Steinhardt, and N. Turok, Local conformal symmetry in physics and cosmology, Phys. Rev. D 89, 043515 (2014).

[10] R. Kallosh and A. Linde, Universality class in conformal inflation, J. Cosmol. Astropart. Phys. 07 (2013) 002.

[11] R. Kallosh and A. Linde, Superconformal generalizations of the Starobinsky model, J. Cosmol. Astropart. Phys. 06 (2013) 028.

[12] R. Kallosh, A. Linde, and D. Roest, Superconformal inflationary $\alpha$-attractors, J. High Energy Phys. 11 (2013) 198. 
[13] R. Kallosh, A. Linde, and D. Roest, Large field inflation and double $\alpha$-attractors, J. High Energy Phys. 08 (2014) 052.

[14] A. Linde, Single-field $\alpha$-attractors, J. Cosmol. Astropart. Phys. 05 (2015) 003.

[15] R. Kallosh and A. Linde, Superconformal generalization of the chaotic inflation model $\frac{\lambda}{4} \phi^{4}-\frac{\xi}{2} \phi^{2} R$, J. Cosmol. Astropart. Phys. 06 (2013) 027.

[16] R. Kallosh and A. Linde, Multi-field conformal cosmological attractors, J. Cosmol. Astropart. Phys. 12 (2013) 006.

[17] S. Ferrara, R. Kallosh, A. Linde, A. Marrani, and A. Van Proeyen, Superconformal symmetry, NMSSM, and inflation, Phys. Rev. D 83, 025008 (2011).

[18] W. Buchmüller, V. Domcke, and K. Schmitz, Superconformal D-term inflation, J. Cosmol. Astropart. Phys. 04 (2013) 019.

[19] W. Buchmuller, V. Domcke, and K. Kamada, The Starobinsky model from superconformal D-term inflation, Phys. Lett. B 726, 467 (2013).

[20] P. Brax and A. Davis, Conformal inflation coupled to matter, J. Cosmol. Astropart. Phys. 05 (2014) 019.

[21] K. Ishiwata, Superconformal subcritical hybrid inflation, Phys. Lett. B 782, 367 (2018).

[22] H. Bernardo, R. Costa, H. Nastase, and A. Weltman, Conformal inflation with chameleon coupling, J. Cosmol. Astropart. Phys. 04 (2019) 027.

[23] F. W. Hehl, J. McCrea, E. W. Mielke, and Y. Ne'eman, Metric affine gauge theory of gravity: Field equations, Noether identities, world spinors, and breaking of dilation invariance, Phys. Rep. 258, 1 (1995).

[24] A. Kleyn, Metric-affine manifold, arXiv:gr-qc/0405028.

[25] V. Vitagliano, T. P. Sotiriou, and S. Liberati, The dynamics of metric-affine gravity, Ann. Phys. (Amsterdam) 326, 1259 (2011); 329, 186(E) (2013).

[26] V. Vitagliano, The role of nonmetricity in metric-affine theories of gravity, Classical Quant. Grav. 31, 045006 (2014).

[27] R. Vazirian, M. Tanhayi, and Z. Motahar, Weyl-invariant extension of the metric-affine gravity, Adv. High Energy Phys. 2015, 902396 (2015).

[28] K. Aoki and K. Shimada, Galileon and generalized Galileon with projective invariance in a metric-affine formalism, Phys. Rev. D 98, 044038 (2018).

[29] F. Bauer and D. A. Demir, Higgs-Palatini inflation and unitarity, Phys. Lett. B 698, 425 (2011).

[30] F. Bauer and D. A. Demir, Inflation with non-minimal coupling: Metric versus Palatini formulations, Phys. Lett. B 665, 222 (2008).

[31] K. Shimada, K. Aoki, and K.-i. Maeda, Metric-affine gravity and inflation, Phys. Rev. D 99, 104020 (2019).

[32] T. Takahashi, T. Tenkanen, and S. Yokoyama, Violation of slow-roll in nonminimal inflation, Phys. Rev. D 102, 043524 (2020).

[33] T. P. Sotiriou and S. Liberati, Metric-affine $f(R)$ theories of gravity, Ann. Phys. (Amsterdam) 322, 935 (2007).

[34] T. P. Sotiriou and V. Faraoni, f(R) theories of gravity, Rev. Mod. Phys. 82, 451 (2010).

[35] T. P. Sotiriou, $f(R)$ gravity, torsion and non-metricity, Classical Quant. Grav. 26, 152001 (2009).
[36] L. Järv, A. Racioppi, and T. Tenkanen, Palatini side of inflationary attractors, Phys. Rev. D 97, 083513 (2018).

[37] T. Helpin and M.S. Volkov, Varying the Horndeski Lagrangian within the Palatini approach, J. Cosmol. Astropart. Phys. 01 (2020) 044.

[38] M. Kubota, K.-Y. Oda, K. Shimada, and M. Yamaguchi, Cosmological perturbations in Palatini formalism, J. Cosmol. Astropart. Phys. 03 (2021) 006.

[39] V.-M. Enckell, K. Enqvist, S. Rasanen, and E. Tomberg, Higgs inflation at the hilltop, J. Cosmol. Astropart. Phys. 06 (2018) 005.

[40] S. Rasanen, Higgs inflation in the Palatini formulation with kinetic terms for the metric, Open J. Astrophys. 2, 1 (2019).

[41] N. Tamanini and C. R. Contaldi, Inflationary perturbations in Palatini generalised gravity, Phys. Rev. D 83, 044018 (2011).

[42] S. Rasanen and P. Wahlman, Higgs inflation with loop corrections in the Palatini formulation, J. Cosmol. Astropart. Phys. 11 (2017) 047.

[43] T. Tenkanen, Resurrecting quadratic inflation with a nonminimal coupling to gravity, J. Cosmol. Astropart. Phys. 12 (2017) 001.

[44] A. Racioppi, Coleman-Weinberg linear inflation: Metric vs. Palatini formulation, J. Cosmol. Astropart. Phys. 12 (2017) 041.

[45] T. Markkanen, T. Tenkanen, V. Vaskonen, and H. Veermäe, Quantum corrections to quartic inflation with a non-minimal coupling: Metric vs. Palatini, J. Cosmol. Astropart. Phys. 03 (2018) 029.

[46] A. Racioppi, New universal attractor in nonminimally coupled gravity: Linear inflation, Phys. Rev. D 97, 123514 (2018).

[47] P. Carrilho, D. Mulryne, J. Ronayne, and T. Tenkanen, Attractor behaviour in multifield inflation, J. Cosmol. Astropart. Phys. 06 (2018) 032.

[48] V.-M. Enckell, K. Enqvist, S. Rasanen, and L.-P. Wahlman, Inflation with $R^{2}$ term in the Palatini formalism, J. Cosmol. Astropart. Phys. 02 (2019) 022.

[49] I. Antoniadis, A. Karam, A. Lykkas, and K. Tamvakis, Palatini inflation in models with an $R^{2}$ term, J. Cosmol. Astropart. Phys. 11 (2018) 028.

[50] S. Rasanen and E. Tomberg, Planck scale black hole dark matter from Higgs inflation, J. Cosmol. Astropart. Phys. 01 (2019) 038.

[51] K. Kannike, A. Kubarski, L. Marzola, and A. Racioppi, A minimal model of inflation and dark radiation, Phys. Lett. B 792, 74 (2019).

[52] J. P. B. Almeida, N. Bernal, J. Rubio, and T. Tenkanen, Hidden inflaton dark matter, J. Cosmol. Astropart. Phys. 03 (2019) 012.

[53] I. Antoniadis, A. Karam, A. Lykkas, T. Pappas, and K. Tamvakis, Rescuing quartic and natural inflation in the Palatini formalism, J. Cosmol. Astropart. Phys. 03 (2019) 005.

[54] H. Azri and D. Demir, Affine inflation, Phys. Rev. D 95, 124007 (2017).

[55] A. Edery and Y. Nakayama, Palatini formulation of pure $R^{2}$ gravity yields Einstein gravity with no massless scalar, Phys. Rev. D 99, 124018 (2019). 
[56] H. Azri and S. Nasri, Entropy production in affine inflation, Phys. Rev. D 101, 064073 (2020).

[57] I. D. Gialamas and A. B. Lahanas, Reheating in $R^{2}$ Palatini inflationary models, Phys. Rev. D 101, 084007 (2020).

[58] A. Karam, S. Karamitsos, and M. Saal, $\beta$-function reconstruction of Palatini inflationary attractors, arXiv: 2103.01182.

[59] I. D. Gialamas, A. Karam, A. Lykkas, and T. D. Pappas, Palatini-Higgs inflation with nonminimal derivative coupling, Phys. Rev. D 102, 063522 (2020).

[60] I. D. Gialamas, A. Karam, and A. Racioppi, Dynamically induced Planck scale and inflation in the Palatini formulation, J. Cosmol. Astropart. Phys. 11 (2020) 014.

[61] T. Tenkanen, Tracing the high energy theory of gravity: An introduction to Palatini inflation, Gen. Relativ. Gravit. 52, 33 (2020).

[62] P. G. Ferreira, C. T. Hill, J. Noller, and G. G. Ross, Scaleindependent $R^{2}$ inflation, Phys. Rev. D 100, 123516 (2019).

[63] Y. Tang and Y.-L. Wu, Inflation in gauge theory of gravity with local scaling symmetry and quantum induced symmetry breaking, Phys. Lett. B 784, 163 (2018).

[64] A. Barnaveli, S. Lucat, and T. Prokopec, Inflation as a spontaneous symmetry breaking of Weyl symmetry, J. Cosmol. Astropart. Phys. 01 (2019) 022.

[65] Y. Tang and Y.-L. Wu, Conformal $\alpha$-attractor inflation with Weyl gauge field, J. Cosmol. Astropart. Phys. 03 (2020) 067.

[66] Y. Tang and Y.-L. Wu, Weyl symmetry inspired inflation and dark matter, Phys. Lett. B 803, 135320 (2020).

[67] D. M. Ghilencea and H. M. Lee, Weyl gauge symmetry and its spontaneous breaking in the standard model and inflation, Phys. Rev. D 99, 115007 (2019).

[68] D. M. Ghilencea, Palatini quadratic gravity: Spontaneous breaking of gauged scale symmetry and inflation, Eur. Phys. J. C 80, 1147 (2020).

[69] D. M. Ghilencea, Gauging scale symmetry and inflation: Weyl versus Palatini gravity, arXiv:2007.14733.

[70] D. M. Ghilencea, Weyl $\mathrm{R}^{2}$ inflation with an emergent Planck scale, J. High Energy Phys. 10 (2019) 209.

[71] D. Iosifidis and T. Koivisto, Scale transformations in metric-affine geometry, Universe 5, 82 (2019).

[72] Y. Mikura, Y. Tada, and S. Yokoyama, Conformal inflation in the metric-affine geometry, Europhys. Lett. 132, 39001 (2020).

[73] C. Armendariz-Picon, T. Damour, and V. F. Mukhanov, k-inflation, Phys. Lett. B 458, 209 (1999).

[74] J. Garriga and V. F. Mukhanov, Perturbations in k-inflation, Phys. Lett. B 458, 219 (1999).

[75] E. Silverstein and D. Tong, Scalar speed limits and cosmology: Acceleration from D-acceleration, Phys. Rev. D 70, 103505 (2004).

[76] N. Arkani-Hamed, H.-C. Cheng, M. A. Luty, and S. Mukohyama, Ghost condensation and a consistent infrared modification of gravity, J. High Energy Phys. 05 (2003) 074.

[77] N. Arkani-Hamed, P. Creminelli, S. Mukohyama, and M. Zaldarriaga, Ghost inflation, J. Cosmol. Astropart. Phys. 04 (2004) 001.
[78] T. Kobayashi, M. Yamaguchi, and J. Yokoyama, G-Inflation: Inflation Driven by the Galileon Field, Phys. Rev. Lett. 105, 231302 (2010).

[79] T. Kobayashi, M. Yamaguchi, and J. Yokoyama, Generalized G-inflation: Inflation with the most general secondorder field equations, Prog. Theor. Phys. 126, 511 (2011).

[80] S. Hashiba and J. Yokoyama, Gravitational reheating through conformally coupled superheavy scalar particles, J. Cosmol. Astropart. Phys. 01 (2019) 028.

[81] X. Chen, M.-x. Huang, S. Kachru, and G. Shiu, Observational signatures and non-Gaussianities of general single field inflation, J. Cosmol. Astropart. Phys. 01 (2007) 002.

[82] N. Aghanim et al. (Planck Collaboration), Planck 2018 results. VI. Cosmological parameters, Astron. Astrophys. 641, A6 (2020).

[83] Y. Akrami et al. (Planck Collaboration), Planck 2018 results. IX. Constraints on primordial non-Gaussianity, Astron. Astrophys. 641, A9 (2020).

[84] M. Hazumi et al., LiteBIRD: A satellite for the studies of b-mode polarization and inflation from cosmic background radiation detection, J. Low Temp. Phys. 194, 443 (2019).

[85] K. N. Abazajian et al. (CMB-S4 Collaboration), CMB-S4 Science Book, 1st ed., arXiv:1610.02743.

[86] K. Abazajian et al. (CMB-S4 Collaboration), CMB-S4: Forecasting constraints on primordial gravitational waves, arXiv:2008.12619.

[87] D. Yamauchi, S. Yokoyama, and K. Takahashi, Multitracer technique for galaxy bispectrum: An application to constraints on nonlocal primordial non-Gaussianities, Phys. Rev. D 95, 063530 (2017).

[88] C. Caprini and D. G. Figueroa, Cosmological backgrounds of gravitational waves, Classical Quant. Grav. 35, 163001 (2018).

[89] F. D'Eramo and K. Schmitz, Imprint of a scalar era on the primordial spectrum of gravitational waves, Phys. Rev. Research 1, 013010 (2019).

[90] L. H. Ford, Gravitational particle creation and inflation, Phys. Rev. D 35, 2955 (1987).

[91] A. A. Starobinsky and J. Yokoyama, Equilibrium state of a selfinteracting scalar field in the De Sitter background, Phys. Rev. D 50, 6357 (1994).

[92] L. Kofman, A. D. Linde, and A. A. Starobinsky, Towards the theory of reheating after inflation, Phys. Rev. D 56, 3258 (1997).

[93] E. Chun, S. Scopel, and I. Zaballa, Gravitational reheating in quintessential inflation, J. Cosmol. Astropart. Phys. 07 (2009) 022.

[94] T. Kunimitsu and J. Yokoyama, Higgs condensation as an unwanted curvaton, Phys. Rev. D 86, 083541 (2012).

[95] H. Tashiro, T. Chiba, and M. Sasaki, Reheating after quintessential inflation and gravitational waves, Classical Quant. Grav. 21, 1761 (2004).

[96] K. Nakayama, S. Saito, Y. Suwa, and J. Yokoyama, Probing reheating temperature of the universe with gravitational wave background, J. Cosmol. Astropart. Phys. 06 (2008) 020.

[97] M. Giovannini, Production and detection of relic gravitons in quintessential inflationary models, Phys. Rev. D 60, 123511 (1999). 
[98] M. Giovannini, Spikes in the relic graviton background from quintessential inflation, Classical Quant. Grav. 16, 2905 (1999).

[99] D. Babusci and M. Giovannini, Sensitivity of wide band detectors to quintessential gravitons, Phys. Rev. D 60, 083511 (1999).

[100] A. Riazuelo and J.-P. Uzan, Quintessence and gravitational waves, Phys. Rev. D 62, 083506 (2000).

[101] M. Sami and V. Sahni, Quintessential inflation on the brane and the relic gravity wave background, Phys. Rev. D 70, 083513 (2004).

[102] M. Artymowski, O. Czerwinska, Z. Lalak, and M. Lewicki, Gravitational wave signals and cosmological consequences of gravitational reheating, J. Cosmol. Astropart. Phys. 04 (2018) 046.
[103] N. Seto, S. Kawamura, and T. Nakamura, Possibility of Direct Measurement of the Acceleration of the Universe Using $0.1-\mathrm{Hz}$ Band Laser Interferometer Gravitational Wave Antenna in Space, Phys. Rev. Lett. 87, 221103 (2001).

[104] S. Kawamura et al., The Japanese space gravitational wave antenna: DECIGO, Classical Quant. Grav. 28, 094011 (2011).

[105] M. Punturo et al., The Einstein telescope: A thirdgeneration gravitational wave observatory, Classical Quant. Grav. 27, 194002 (2010).

[106] R. H. Cyburt, B. D. Fields, K. A. Olive, and T.-H. Yeh, Big Bang Nucleosynthesis: 2015, Rev. Mod. Phys. 88, 015004 (2016). 